\title{
A spatially extended model for macroscopic spike-wave discharges
}

\author{
Peter Neal Taylor • Gerold Baier
}

Received: 4 March 2011 / Revised: 30 March 2011 / Accepted: 11 April 2011 / Published online: 10 May 2011

(C) Springer Science+Business Media, LLC 2011

\begin{abstract}
Spike-wave discharges are a distinctive feature of epileptic seizures. So far, they have not been reported in spatially extended neural field models. We study a space-independent version of the Amari neural field model with two competing inhibitory populations. We show that this competition leads to robust spikewave dynamics if the inhibitory populations operate on different time-scales. The spike-wave oscillations present a fold/homoclinic type bursting. From this result we predict parameters of the extended Amari system where spike-wave oscillations produce a spatially homogeneous pattern. We propose this mechanism as a prototype of macroscopic epileptic spike-wave discharges. To our knowledge this is the first example of robust spike-wave patterns in a spatially extended neural field model.
\end{abstract}

Keywords Epilepsy • EEG • Mathematical modelling • Spike-wave $\cdot$ Bursting

\section{Action Editor: Alain Destexhe}

Source code for simulations is available at http://senselab. med.yale.edu/modeldb/.

Electronic supplementary material The online version of this article (doi:10.1007/s10827-011-0332-1) contains supplementary material, which is available to authorized users.

P. N. Taylor $(\varangle) \cdot$ G. Baier Manchester Interdisciplinary Biocentre,

The University of Manchester, Manchester, M1 7DN, UK

e-mail: peter.taylor@postgrad.manchester.ac.uk

\section{Introduction}

Spike-wave discharges (SWD) are a striking nonlinear phenomenon in electroencephalogram (EEG) which occur frequently in epileptic disorders, especially in children, and are not well-understood in terms of its spatio-temporal features (for a review see Blumenfeld 2005). SWD are known to be electrographical correlates of generalised epileptic seizures, specifically absence seizures (Sadleir et al. 2006) but also other types like myoclonic seizures (Asconapé and Penry 1984), seizures of the Lennox-Gastaut type (see e.g. Aicardi 1988), the clonic part of primary generalised tonic clonic seizures, and are also seen during the secondary generalisation of complex partial seizures (for EEG features see e.g. Stern and Engel 2005). Independent of the seizure type, SWD are viewed as a fundamental pathological behavior of hypersynchronised neural populations at the macroscopic level (McCormick and Contreras 2001) which present a classic example of selforganised nonlinear dynamics (da Silva et al. 2003).

Traditionally SWD were considered to be periodic homogeneous oscillations on a macroscopic spatial scale. Previous mathematical modelling approaches therefore focussed exclusively on space independent systems, using a small number of variables and neglecting the spatial characteristics. For the case of absence seizures, Suffczynski et al. proposed a mesoscale model which incorporated a mechanism of bistability (Suffczynski et al. 2004). Robinson et al. proposed a differently motivated thalamocortical model with explicit time delays to produce SWD (Robinson et al. 2002; Breakspear et al. 2006). More recently Marten et al. (2009a, b) incorporated two separate inhibitory mechanisms in a modified version of this model and 
reported the generation of spike-wave and polyspikewave oscillations due to the different time scales of $\mathrm{GABA}_{A}$ and $\mathrm{GABA}_{B}$ inhibition. This was based on a previous detailed neuronal model (Destexhe 1998; Destexhe and Sejnowski 2001) which included inhibitory $\mathrm{GABA}_{A}$ and $\mathrm{GABA}_{B}$ receptor mediated firing operating on two different inhibitory time scales. The authors of Wendling et al. (2002) also assumed two inhibitory populations operating at different time scales to account for the transition to epileptic spiking, but not SWD, in a mesoscopic model of temporal lobe epilepsy.

However, mounting evidence suggests that there are important spatial components in the so-called generalised seizures with SWD. This has in particular led to a discussion about the potentially focal onset of allegedly primary generalised seizures with rapid secondary generalisation (Holmes et al. 2004; Holmes 2008). Evidence for this hypothesis comes from the electrophysiological observation of focal cortical onset of SWD in a genetic rat model of absence seizures (Meeren et al. 2002; van Luijtelaar and Sitnikova 2006). A combination of EEG with fMRI studies led Blumenfeld to propose specifically heterogeneous thalamocortical networks involved in SWD generation (Blumenfeld 2005). The author proposed that an improved understanding of the heterogeneous brain regions involved might lead to a more effective treatment of spike-wave seizures. In humans electrographic studies (Rodin and Ancheta 1987), time series analysis (Amor et al. 2005), EEG and MEG source localisation (Westmijse et al. 2009) and combined EEG/fMRI imaging studies (Moeller et al. 2008; Bai et al. 2010) have revealed distinct spatial features of SWD presumably arising from local cortical (or indeed thalamocortical) networks, specifically including frontal and parietal areas.

In this letter, we simplify the classical Amari neural field model (Amari 1977) to a low dimensional ODE and show that an extension of this ODE (similar to the one used in Wendling et al. 2002) can generate spike-wave discharges. This is achieved with a mechanism of deterministic bursting, defined as the repetitive alternation between fast spiking and a quiescent phase (the "wave") (Izhikhevich 2000). If this bursting ODE is then converted back to a neural field equation with three neural populations, spatially homogeneous spike wave solutions are found for the first time in such equations. Note that the clinical use of the term "spike" refers to a specific EEG morphology (i.e. a macroscopic phenomenon), not to the action potential in single neurons. We propose our model as a prototype for robust macroscopic SWD.

\section{ODE model}

We start with an oscillatory model with two variables based on the spatially extended neural field model proposed in Amari (1977). The oscillator is constructed in analogy with the Wilson-Cowan (WC) oscillator in Borisyuk and Kirillov (1992). It is a two variable system consisting of one excitatory and one inhibitory variable and is capable of a (dynamic) instability to generate periodic oscillations (see below). Following Wendling et al. (2002), we expand this model by a second inhibitory variable (or population in the context of neural mass modelling) and assume it to operate at a time scale that is slower than that of the original inhibitory population. The resulting three variable ODE is given by:

$$
\begin{aligned}
& \dot{E}(t)=h_{1}-E+w_{1} f[E]-w_{2} f\left[I_{1}\right]-w_{3} f\left[I_{2}\right] \\
& \dot{I}_{1}(t)=\left(h_{2}-I_{1}+w_{4} f[E]\right) / \tau_{1} \\
& \dot{I}_{2}(t)=\left(h_{3}-I_{2}+w_{5} f[E]\right) / \tau_{2}
\end{aligned}
$$

Where $w_{1 . .5}$ are connectivity parameters, $\tau_{1,2}$ are time scale parameters and $h_{1 \ldots 3}$ are analogous to the additive constants used in the original Amari model. The piecewise linear (PWL) function $(f)$ is $f=0$ if $v \leq-l$; $f=(v+l) / 2 l$ if $-l<v<l$; and $f=1$ if $v \geq l$, where $l>0$ determines the steepness of the transition and $v=$ $E, I_{1}$ or $I_{2}$. This replaces the Heaviside step function in the original Amari model and has been used by other authors since Kilpatrick and Bressloff (2010). In addition, it was shown that the Heaviside function does not permit spike-wave-solution in a mean-field model of SWD (Rodrigues et al. 2006). Qualitatively similar results as the ones presented here can, in addition, also be obtained with a smooth sigmoid function as used by other authors (Jansen and Rit 1995, and see Eq. (3) below). Incidentally, SWD dynamics can also be found in the Wilson-Cowan oscillator (Wilson and Cowan 1972) extended by a slow inhibitory variable and also the Jansen and Rit model extended by a pair of variables representing a slow inhibitory population (Goodfellow et al. 2011).

\section{Results}

Figure 1(a) shows a bifurcation diagram of the fast two variable subsystem $\left(E, I_{1}\right)$ in Eq. (1) with $w_{3}=0$, scanning parameter $h_{1}$. Limit cycle oscillations around an unstable focus are found as the only attracting solution in the displayed region as long as $h_{1}<-0.05$. As $h_{1}$ is increased the limit cycle disappears at $h_{1} \approx 0.1$ in a homoclinic bifurcation (labelled Crit. 1) caused by its collision with the stable manifold of the saddle 
(a)

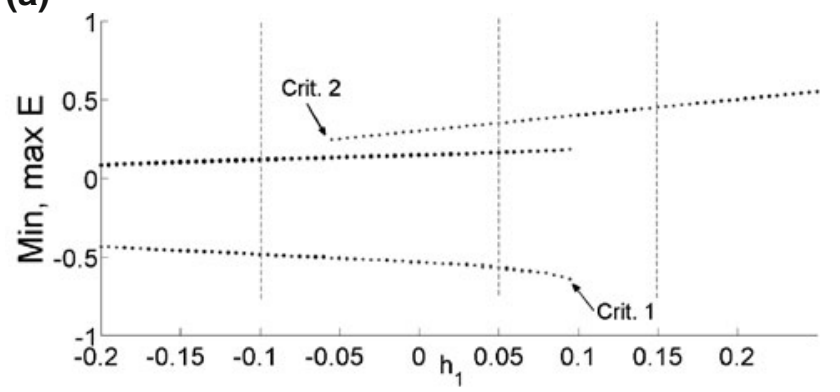

(b)

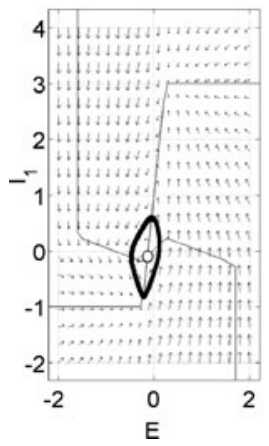

(c)

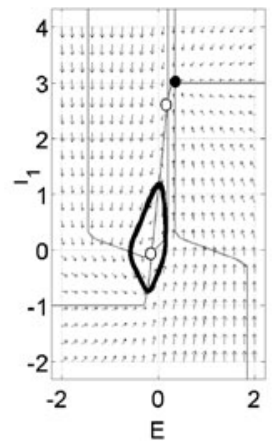

(d)

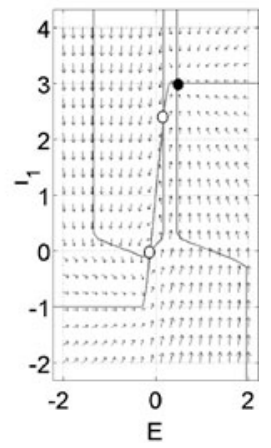

Fig. 1 (a) Bifurcation diagram of the fast subsystem $E, I_{1}$ in Eq. (1) with $w_{3}=0$. Crit. 1 and Crit. 2 refer to bifurcation points. The vertical dotted lines indicate the parameter values for which the corresponding phase space diagrams are plotted in panels (b) - (d). (b) - (d) Phase space representation of the vector fields at distinct points of the bifurcation diagram in (a). (b) $h_{1}=-0.1$; (c) $h_{1}=0.05$; and (d) $h_{1}=0.15$. Continuous lines are nullclines. Filled (open) circles denote stable (unstable) fixed points. Bold black line is the stable limit cycle. The stable fixed point is a node. The unstable fixed points are a focus and saddle, respectively. A complete table of parameters for all figures is provided as supporting online material

(compare Fig. 1(c) and (d)). Beyond the homoclinic bifurcation the only attractor is a stable node (see Fig. 1(d)). If, starting from values where only the stable node exists, parameter $h_{1}$ is decreased, the critical situation labelled Crit. 2 is reached where the node disappears in a collision with the saddle (a fold bifurcation, compare Fig. 1(c) and (b)).

The bistable region $\left(-0.05<h_{1}<0.1\right)$ fulfils the criteria to generate bursting of type "fold/homoclinic" as classified in Izhikhevich (2000). Thus, this type of bursting (sometimes also referred to as "type 1" or "square wave" bursting) can now be generated by including the slow third variable to this two variable system. When the third variable is included $\left(w_{3}>0\right)$, the full model can display an autonomous transition from the fast oscillatory state to the non-oscillatory state and back again which apparently shifts parameter $h_{1}$ periodically between the monostable oscillatory region $\left(h_{1}<-0.05\right)$ and the monostable steady state region $\left(h_{1}>0.1\right)$. With proper adjustment of the slow time scale of the third variable the resulting dynamics displays only one spike while in the oscillatory region, resulting in globally stable spike-wave discharges. Figure 2(a) shows a bifurcation diagram of a scan in the $\left(h_{1}, h_{2}, h_{3}\right)$ parameter space, where solutions with SWD are displayed. The SWD region is found over a wide range of parameter sets and is thus a robust behaviour of Eq. (1). In the coloured version of the figure (online only) it can be seen that in addition to single-spike wave dynamics, there is also a region of multiple-spikewave discharges (two and three spikes, respectively). This refers to the occurrence of multiple fast oscillatory spikes between pseudo-steady state waves. In clinical neurology, this is commonly referred to as polyspikewave discharges (pSWD).

A bifurcation structure that is qualitatively similar to the one displayed in Fig. 2(a) (i.e. including SWD and pSWD) is also found for other values of the time scale parameters, connectivity parameters, and the parameters of the function $f$. In addition, it is found that due to the symmetry of the PWL function $f$, a second pair of fixed points equivalent to the saddle and node in Fig. 1(c) and (d) is found for more negative values of $h_{2}$ (roughly between -3.5 and -4 ) which allows for a second region of SWD similar to the one displayed in Fig. 2(a). Importantly, we find that the region of single spike-wave discharges is wide in the $h_{1}$ direc-
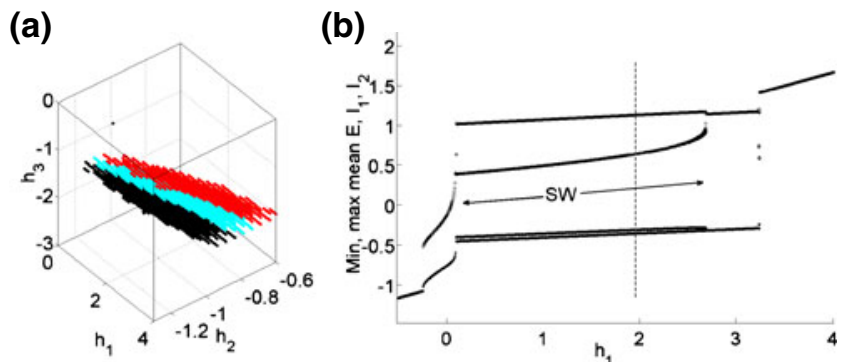

(c)

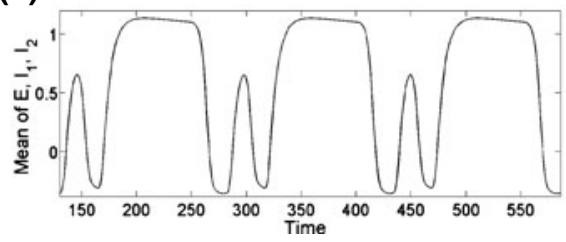

(d)

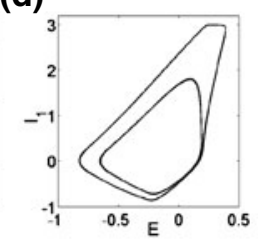

Fig. 2 (a) Scan of $h_{1}, h_{2}, h_{3}$ parameter space. SWD regions are indicated by black dots. In the colour version (online only) cyan dots indicate 2 -spike-wave, red spots indicate 3 -spike-wave. (b) Bifurcation diagram scanning $h_{1}$ where $h_{2}=-1$ and $h_{3}=-2$. (c) Time series showing SWD in the mean of all populations using $h_{1}=2$ (dashed line in (b), other parameters as in (b)). (d) Projection of spike-wave trajectory in (c) onto the $E / I_{1}$ state space 
tion as displayed in Fig. 2(b). Figure 2(b) also shows that the SWD region is bracketed by small amplitude oscillations for smaller $h_{1}$ and simple large amplitude oscillations and fixed point dynamics for larger $h_{1}$. The transition from large ampitude oscillations at $h_{1} \approx 2.7$ is due to a false bifurcation (i.e. a smooth deformation of the limit cycle as studied by Rodrigues et al. (2010)).

The model presented thus far is a spatially homogeneous ODE version of the original Amari model with the addition of a second inhibitory population operating at a different time scale and with the use of a PWL function.

If we now include a spatial extension in the form of "Mexican hat" connectivity as in the original Amari model we can reconstruct a modified version of the Amari equation which includes the second inhibitory population:

$$
\begin{aligned}
\dot{E(i)}= & h_{1}-E(i)+\sum_{j=1 \ldots n} w_{1}(i-j) f[E(j)] \\
& -\sum_{j=1 \ldots n} w_{2}(i-j) f\left[I_{1}(j)\right] \\
& -\sum_{j=1 \ldots n} w_{3}(i-j) f\left(\left[I_{2}(j)\right]\right. \\
\dot{I_{1}(i)=} & \left(h_{2}-I_{1}(i)+w_{4} f[E(i)]\right) / \tau_{1} \\
\dot{I_{2}(i)=} & \left(h_{3}-I_{2}(i)+w_{5} f[E(i)]\right) / \tau_{2}
\end{aligned}
$$

The Mexican hat connectivity functions $\left(w_{1 \ldots 5}\right)$ are identical to those used by Amari, $n$ is the number of spatial locations. The connectivities of the slow inhibitory population are implemented in analogy to those of the fast inhibitory population. Simulations of Eq. (2) were performed with periodic boundary conditions for one and two spatial dimensions.

SWD was found to be present for many different combinations of connectivity values. This is shown in the bifurcation diagram Fig. 3(a) scanning $h_{1}$ in the case of one spatial dimension. For large values of $h_{1}$ a fixed point can be observed, upon decreasing $h_{1}$ to the range $3<h_{1}<6.75$ stable spike-wave oscillations occur which are synchronised across the whole field (labelled "S-W"). Decreasing $h_{1}$ further results in the break up of the synchronisation before the appearance of a two-spike wave solution reminiscent of the pSWD found in Eq. (1) (labelled in cyan in Fig. 2(a)). This is denoted as " $2 \mathrm{~S}-\mathrm{W}$ ". Decreasing $h_{1}$ further, results in desynchronised and synchronised oscillations, respectively, before the appearance of another fixed point with low values of the mean. Figure 3(a) was produced using the PWL function and is thus comparable to Fig. 2(b). Figure 3(b) (top and middle) is an exemplary

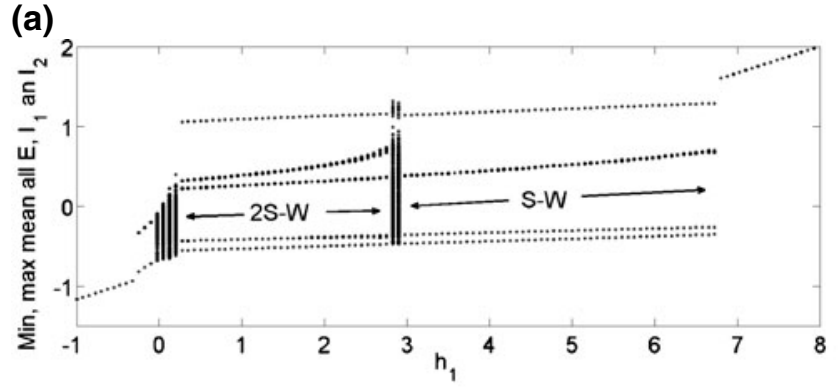

(b)
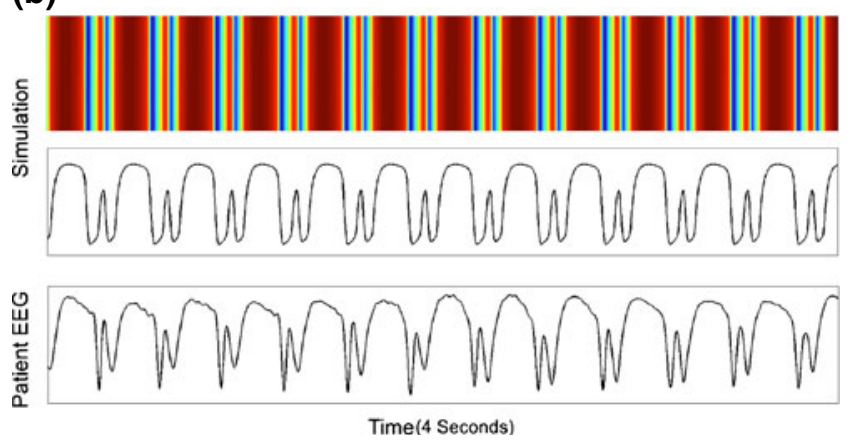

Fig. 3 (a) Bifurcation diagram showing extrema of the mean of all locations in the spatially extended 1D model (2) using the PWL function. Scanning $h_{1}$ yields a region of synchronised SWD (between 3 and 6.75) and 2-SWD (between 0.3 and 2.8). (b) Upper panel (in colour online): time series of synchronised SWD output of the excitatory population in Eq. (2) for $h_{1}=5$ in one spatial dimension with sigmoid transfer function (3). Middle: time series of the mean of all populations in all locations. Same simulation as the upper panel. Lower: $4 \mathrm{~s}$ of an EEG recording of a patient with absence epilepsy taken from electrode C3

time series of the one dimensional system using a sigmoid (Eq. (3)) rather than the PWL function.

$f\left(E, I_{1,2}\right)=\frac{1}{1+\varsigma^{-E, I_{1,2}}}$

The figures show the highly synchronised activity of the spatially extended system. The time series are nearly identical for both PWL and sigmoid version, confirming the robustness of this solution. The only difference is that the former "square" shape of the wave gives way to a more rounded wave component. Further to this, the mean of all populations in all locations shows the distinctive spike wave profile observed in the one location study. This mean waveform is highly reminiscent of the waveform in the clinically recorded EEG, an example of which is shown in Fig. 3(b) (bottom). In Fig. 3(a), the SWD solution immediately borders the fixed point solution for large values of $h_{1}$. We thus have a situation comparable to the one used in Breakspear et al. (2006) and Marten et al. (2009a) to describe transitions to absence seizure in a space-independent model. In addition, we also found a transition from fast 
low-amplitude oscillations to SWD for some values of $h_{2}$ which is relevant as some authors consider cortical background to be noisy fast oscillations (e.g. Jansen and Rit 1995 and Wendling et al. 2002). Qualitatively similar results of robust SWD and pSWD with the above mentioned transitions were found in simulations of the model with two spatial dimensions (results not shown). All reported results were found to be stable in the presence of added independent noise in all locations. Thus, our model (3) allows to explain the transition to SWD in the same manner as previous approaches, except that we now have an explicit representation of cortical locations.

\section{Discussion}

Perhaps the most striking property of typical absence seizure EEG recordings is the high degree of spatiotemporal synchronisation of the spike and wave dynamics. This, along with its characteristic waveform, suggests the possibility of an underlying low dimensional dynamic mechanism being the cause despite the high dimensional complexity of resting state background EEG. To generate SWD we have used a mechanism based on two inhibitory timescales similar to what was introduced in previous modelling studies (Destexhe 1998; Marten et al. 2009a, b; Wendling et al. 2002). The time courses of various inhibitory synaptic processes in the neocortex have been shown to be highly variable and span more than one order of magnitude (Thomson and Deuchars 1997; Otis and De Koninck 1993). However, the origin of the slow timescale resulting from $\mathrm{GABA}_{B}$ receptor activation has recently been challenged (Bazhenov et al. 2008). It is therefore possible that the slow process might also be attributed to non-synaptic mechanisms which our phenomenological model can also account for.

An indispensable requirement for a prototypic model of spike wave is that its dynamics is robust and can be found over a wide range of parameter space because absence seizures tend to occur frequently throughout the day, i.e. under varying environmental conditions. This is found to be the case both in our lowdimensional prototype (Eq. (1)) and in the extended Amari model (2) in one and two spatial dimensions.

Given that spike wave oscillations are generally thought to arise from both corticocortical and thalamocortical interactions rather than cortical interactions alone, a future study should now include thalamocortical interactions to relate more closely to electrophysiological knowledge (McCormick and Contreras 2001). This can be done starting from the three variable model (2). The model (2) uses the widely used classical Amari model as a basis and can now be extended further to account for observed heterogeneities and fragmentation of absence SWD or the more irregular generalised SWD of myoclonic seizures or the LennoxGastaut syndrome (Sadleir et al. 2006). Heterogeneity in the sense of patchy long range connections can be modelled more realistically by using connectivity estimates from diffusion weighted magnetic resonance imaging (DW-MRI) of neocortical tissue (Hagmann et al. 2007). This will then lead to a possible mechanistic explanation of recent observations like localised microseizures in normal and epileptic neocortical tissue (Stead et al. 2010) and to predictions of the spatiotemporal responses to local electric stimuli in humans (David et al. 2010).

To summarise, we have demonstrated epileptic spike-wave discharges in an extended version of Amari's neural field model. A slow inhibitory population robustly generates SWD via a bursting mechanism in a region of bistability of a fast oscillatory subsystem. This is found both in a space independent ODE and also in the spatially extended case. This has not been reported thus far in spatially extended neural field models at the macroscopic scale. Our model therefore serves as a prototypic equation for future modelling of clinical epileptic dynamics in time and space.

Acknowledgements We thank H. Muhle, M. Siniatchkin and U. Stephani, Kiel, for clinical EEG data. We acknowledge financial support form EPSRC and BBSRC. We thank Kaspar Schindler, John Terry, Marc Goodfellow, Yujiang Wang and David Broomhead for discussion.

\section{References}

Aicardi, J. (1988). Epileptic syndromes in childhood. Epilepsia, 29(Suppl 3), S1-5.

Amari, S. (1977). Dynamics of pattern formation in lateralinhibition type neural fields. Biological Cybernetics, 27(2), 77-87.

Amor, F., Rudrauf, D., Navarro, V., N'diaye, K., Garnero, L., Martinerie, J., et al. (2005). Imaging brain synchrony at high spatio-temporal resolution: Application to MEG signals during absence seizures. Signal Processing, 85(11), 21012111.

Asconapé, J., \& Penry, J. K. (1984). Some clinical and eeg aspects of benign juvenile myoclonic epilepsy. Epilepsia, 25(1), 108114.

Bai, X., Vestal, M., Berman, R., Negishi, M., Spann, M., Vega, C., et al. (2010). Dynamic time course of typical childhood absence seizures: Eeg, behavior, and functional magnetic resonance imaging. Journal of Neuroscience, 30(17), 58845893.

Bazhenov, M., Timofeev, I., Fröhlich, F., \& Sejnowski, T. J. (2008). Cellular and network mechanisms of electrographic 
seizures. Drug Discovery Today. Disease models, 5(1), 4557.

Blumenfeld, H. (2005). Cellular and network mechanisms of spike-wave seizures. Epilepsia, 46(Suppl 9), 21-33.

Borisyuk, R. M., \& Kirillov, A. B. (1992). Bifurcation analysis of a neural network model. Biological Cybernetics, 66(4), 319325 .

Breakspear, M., Roberts, J. A., Terry, J. R., Rodrigues, S., Mahant, N., \& Robinson, P. A. A. (2006). Unifying explanation of primary generalized seizures through nonlinear brain modeling and bifurcation analysis. Cerebral Cortex, 16, 1296-1313.

David, O., Bastin, J., Chabardes, S., Minotti, L., \& Kahane, P. (2010). Studying network mechanisms using intracranial stimulation in epileptic patients. Frontiers in Systems Neuroscience, $4,12$.

Destexhe, A. (1998). Spike-and-wave oscillations based on the properties of GABAB receptors. Journal of Neuroscience, 18(21), 9099.

Destexhe, A., \& Sejnowski, T. J. (2001). Thalamocortical assemblies. Oxford: Oxford University Press.

da Silva, F. L., Blanes, W., Kalitzin, S. N., Parra, J., Suffczynski, P., \& Velis, D. (2003). Epilepsies as dynamical diseases of brain systems: Basic models of the transition between normal and epileptic activity. Epilepsia, 44, 72-83.

Goodfellow, M., Schindler, K., \& Baier, G. (2011). Intermittent spike-wave dynamics in a heterogeneous, spatially extended neural mass model. NeuroImage, 55(3), 920-932.

Hagmann, P., Kurant, M., Gigandet, X., Thiran, P., Wedeen, V. J., Meuli, R., et al. (2007). Mapping human whole-brain structural networks with diffusion MRI. PloS ONE, 2(7), e597.

Holmes, M. D. (2008). Dense array EEG: Methodology and new hypothesis on epilepsy syndromes. Epilepsia, 49(Suppl 3), 314.

Holmes, M. D., Brown, M., \& Tucker, D. M. (2004). Are "generalized" seizures truly generalized? Evidence of localized mesial frontal and frontopolar discharges in absence. Epilepsia, 45(12), 1568-1579.

Izhikhevich, E. (2000). Neural excitability, spiking and bursting. International Journal of Bifurcation and Chaos, 10(6), 11711266.

Jansen, B. H., \& Rit, V. G. (1995). Electroencephalogram and visual evoked potential generation in a mathematical model of coupled cortical columns. Biological Cybernetics, 73, 357 366

Kilpatrick, Z. P., \& Bressloff, P. C. (2010). Spatially structured oscillations in a two-dimensional excitatory neuronal network with synaptic depression. Journal of Computational Neuroscience, 28(2):193-209.

Marten, F., Rodrigues, S., Benjamin, O., Richardson, M. P., \& Terry, J. R. (2009a). Onset of polyspike complexes in a mean-field model of human electroencephalography and its application to absence epilepsy. Philosophical Transactions of the Royal Society of London, Series A: Mathematical and Physical Sciences, 367(1891), 1145-1161.

Marten, F., Rodrigues, S., Suffczynski, P., Richardson P. M., \& Terry, J. R. (2009b). Derivation and analysis of an ordinary differential equation mean-field model for studying clinically recorded epilepsy dynamics. Physical Review E (Statistical, Nonlinear, and Soft Matter Physics), 79(2), 21911.
McCormick, D. A., \& Contreras, D. (2001). On the cellular and network bases of epileptic seizures. Annual Review of Physiology, 63, 815-846.

Meeren, H. K. M., Pijn, J. P. M., Van Luijtelaar, E. L. J. M., Coenen, A. M. L., \& Lopes da Silva, F. H. (2002). Cortical focus drives widespread corticothalamic networks during spontaneous absence seizures in rats. Journal of Neuroscience, 22(4), 1480-1495.

Moeller, F., Siebner, H. R., Wolff, S., Muhle, H., Granert, O., Jansen, O., et al. (2008). Simultaneous EEG-fMRI in drug-naive children with newly diagnosed absence epilepsy. Epilepsia, 49(9), 1510-1519.

Otis, T. S., \& De Koninck, Y. (1993). Characterization of synaptically elicited GABAB responses using patch-clamp recordings in rat hippocampal slices. Journal of Physiology, 463, 391-407.

Robinson, P. A., Rennie, C. J., \& Rowe, D. L. (2002). Dynamics of large-scale brain activity in normal arousal states and epileptic seizures. Physical Review E, 65(4), 41924.

Rodin, E., \& Ancheta, O. (1987). Cerebral electrical fields during petit mal absences. Electroencephalography and Clinical Neurophysiology, 66(6), 457-466.

Rodrigues, S., Barton, D., Marten, F., Kibuuka, M., Alarcon, G., Richardson, M. P., et al. (2010). A method for detecting false bifurcations in dynamical systems: Application to neuralfield models. Biological Cybernetics, 102(2), 145-154.

Rodrigues, S., Terry, J. R., \& Breakspear, M. (2006). On the genesis of spike-wave oscillations in a mean-field model of human thalamic and corticothalamic dynamics. Physics Letters A, 355(4-5), 352-357.

Sadleir, L. G., Farrell, K., Smith, S., Connolly, M. B., \& Scheffer, I. E. (2006). Electroclinical features of absence seizures in childhood absence epilepsy. Neurology, 67(3), 413-418.

Stead, M., Bower, M., Brinkmann, B. H., Lee, K., Marsh, W. R., Meyer, F. B., et al. (2010). Microseizures and the spatiotemporal scales of human partial epilepsy. Brain, 133(9), 27892797.

Stern, J. M., \& Engel, J. (2005). Atlas of EEG patterns. Philadelphia: Lippincott Williams \& Wilkins.

Suffczynski, P., Kalitzin, S., \& Lopes Da Silva, F. H. (2004). Dynamics of non-convulsive epileptic phenomena modeled by a bistable neuronal network. Neuroscience, 126(2), 467484.

Thomson, A. M., \& Deuchars, J. (1997). Synaptic interactions in neocortical local circuits: Dual intracellular recordings in vitro. Cerebral Cortex, 7(6), 510-522.

van Luijtelaar, G., \& Sitnikova, E. (2006). Global and focal aspects of absence epilepsy: The contribution of genetic models. Neuroscience and Biobehavioral Reviews, 30(7), 9831003.

Wendling, F., Bartolomei, F., Bellanger, J. J., \& Chauvel, P. (2002). Epileptic fast activity can be explained by a model of impaired GABAergic dendritic inhibition. European Journal of Neuroscience, 15(9), 1499-1508.

Westmijse, I., Ossenblok, P., Gunning, B., \& van Luijtelaar, G. (2009). Onset and propagation of spike and slow wave discharges in human absence epilepsy: A MEG study. Epilepsia, 50(12), 2538-2548.

Wilson, H. R., \& Cowan, J. D. (1972). Excitatory and inhibitory interactions in localized populations of model neurons. Biophysical Journal, 12(1), 1-24. 